\title{
Evidence for climate variations induced by the 11-year solar and cosmic rays cycles
}

\author{
William Bruckman ${ }^{1}$ and Elio Ramos ${ }^{2}$ \\ ${ }^{1}$ Department of Physics and Electronics, University of Puerto Rico at Humacao, \\ CUH Station, 100 Route 908, Humacao, PR 00791-4300 \\ email: miguelwillia.bruckman@upr.edu \\ ${ }^{2}$ Department of Mathematics, University of Puerto Rico at Humacao, \\ CUH Station, 100 Route 908, Humacao, PR 00791-4300 \\ email: elio.ramos@upr.edu
}

\begin{abstract}
We analyzed data from PSMSL monthly mean sea level seeking correlations between sea level fluctuations and the solar and cosmic rays 11 year cycle. The data reveals decadal variability that could be causally connected to the solar and cosmic rays cycle, since these periodic changes are correlated. It is also found that the solar (cosmic rays) cycle correlates (anti-correlates) with the mean global surface temperature anomaly. A probable explanation of the above correlations is that the solar intensity and cosmic rays variations induce oscillations in the average temperature and precipitation, with corresponding changes in the continental water and snow accumulation. Thus, for instance, a higher than average snow and water over land, and lower temperatures produce oceans thermal contraction and lower mass, implicating lower mean sea level.
\end{abstract}

Keywords. Sun: activity, solar-terrestrial relations, cosmic rays

The relevance of the Solar Cycle on Earth climate became an important issue with the publication of an article by J.A. Eddy in the Journal Science in 1976. In this research strong evidence was presented about an historical period between 1645 and 1715, called The Maunder Minimum, where solar-spots virtually disappeared, coinciding this with much lower than average temperatures. The absence of solar-spots during the Maunder minimum was mentioned by Wolf $(1856,1868)$ and Sporer $(1887)$. The investigations was continued by Maunder (1922), who strongly defended the observational evidence from the skeptical scientific community that questioned the reliability of the observations. Eddy was able to convince many that Maunders arguments were sound and robust, and he added new observational evidence, like the absence of solar corona and variations of the Carbon 14 isotopes in the atmosphere, during the Maunder minimum. Carbon 14 production in the atmosphere is sensitive to the intensity of the galactic cosmic rays flux (GCRF) which in turn is modulated by the solar activity cycle. Thus, the solar cycle have an associated GCRF cycle, that is almost perfectly anti-correlated to it.There is also evidence that the GCRF cycle and other solar irradiance indirect influence in the atmosphere affect climate, and we will briefly discussed this topic later on.

Modern measurements by artificial satellites demonstrate indeed, as suggested by the Maunder minimum and similar historical events, that during the solar cycle we have a variation of about $0.1 \%$ in the solar flux, which is very highly correlated with the solarspot 11 year cycle. Although the variation is small (about 1 watt per square meter per cycle) we expect it to have a measurable effect on the Earth surface temperature, and we can see in Fig. 1 that the data of anomalies in sea surface temperature, since the beginning of the NOAA, NCDC record in the year 1880, show oscillations with periodicity 
seemingly in correlation with the solar cycle. To put that interpretation to a statistical test an analysis of the data was carried out, and we found that a cross-correlation function analysis between solar-spot number and the temperature global anomalies of Fig. 1 reveals a significant correlation of $\mathrm{r}=0.64$, with a delay of about 1 year, using the data since 1950. There is a gradual deterioration of the correlation when data before 1950 is included. This reduction in correlation is to be expected if the number of quality measurements decrease with old data. Furthermore, during the interval 1880 to 1940 solar-spot activity was much lower than afterward, thus making perhaps solar variation effects less perceptible.

There is also indirect effects on climate coming from the ways in which the solar flux variations in the ultraviolet can affect temperature in the stratosphere (Haigh 1996, 1999, Schindell et al. 1999). Furthermore, another source for climate alteration could be the formation of ions by GCRF, that, as me mentioned earlier, is a flux modulated by the solar cycle. In fact there is observational evidence (Svensmark and Friis-Chritensen, 1997; Svensmark, 1998) for increments in cloud cover during GCRF maximum(solar-spot minimum), and also Kniveton and Todd (2001) correlated precipitation with GCRF. The above considerations indicate that temperatures can be affected by other mechanism besides direct solar radiation absorption at the surface of the Earth.

Another interesting consequence of solar flux and temperature oscillations is the variation of the mean sea level (MSL), resulting from the thermal contraction and expansion of the oceans and the variation in the accumulation of snow in the continents. We can estimate this sea perturbation by noticing that in about a 50 years interval we have an increase in MSL of approximately $100 \mathrm{~mm}$ (Jevrejeva et al. 2008), while we also have a corresponding change in mean sea surface temperature of near $0.4 \mathrm{~K}$ (NOAA, NCDC, global ocean temperature, in http://climexp.kmni.nl/selectindex.cgi). This information allows us to parametrized MSL change with temperature variation, and thus obtain $\mathrm{dMSL} / \mathrm{dT} \sim 100 \mathrm{~mm} / 0.4 \mathrm{~K}=260 \mathrm{~mm} / \mathrm{K}$. Therefore, for amplitude change in temperature according to Fig. 1 we should have discernible changes in MSL,since MSL variations of even few millimeters are within the observable capability of the permanent system for MSL(PSMSL), and we will describe below MSL variations of the expected magnitude and period correlated with the solar cycle.

A typical time series from PSMSL is shown in Fig. 2, from Magueyes station in Puerto Rico, where we can see a seasonal variation in MSL with a yearly minimum of variable amplitude. These minima are the result of the thermal contraction due to minima in winter sea surface temperature (that happened there around the beginning of march) plus the accumulation of more snow in the continents. We also note that this amplitude have a variation with a periodicity (triangles) similar to the 11 year sunspot cycle. In fact we found a high correlation of 0.71 between the curve define by the minima from Magueyes and the solar cycle, with a delay of 3 years, with similar results for San Juan P.R.(Bruckman and Ramos 2003, 2004, 2007), and individual stations in the Caribbean, and, collectively, in the North Atlantic,North Pacific, and in 1023 stations around the world (Fig. 3). The correlation of global MSL variations, in Fig. 3, with the solar cycle was $\mathrm{r}=0.64$, with a delay of 4 years, for data after 1940 . We also find a lowering in correlation when including data before 1940, similar to what we found for global surface temperature. The explanation of the observed delays require the study of complicated cause and effect inertial time lag. However, for thermal inertia of the oceans we have an analogous delay in the seasonal variation in temperature, where the minimum in temperature do not coincide with the beginning of the winter in the hemisphere. The thermal time lag value depends of thermodynamics parameters and the frequency of the oscillations. 


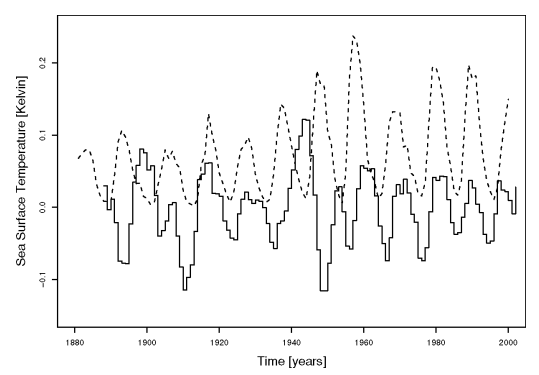

Figure 1. Detrended 3 years running mean (3RN) Sea Surface Temperature (solid) vs. Solar-spot Cycle (dash).

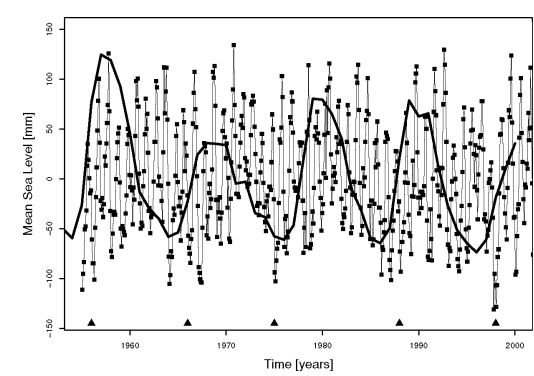

Figure 2. Detrended Mean Sea Level (MSL) for Magueyes, Puerto Rico vs. Solar Cycle (solid). The black triangles point to dipping in MSL.

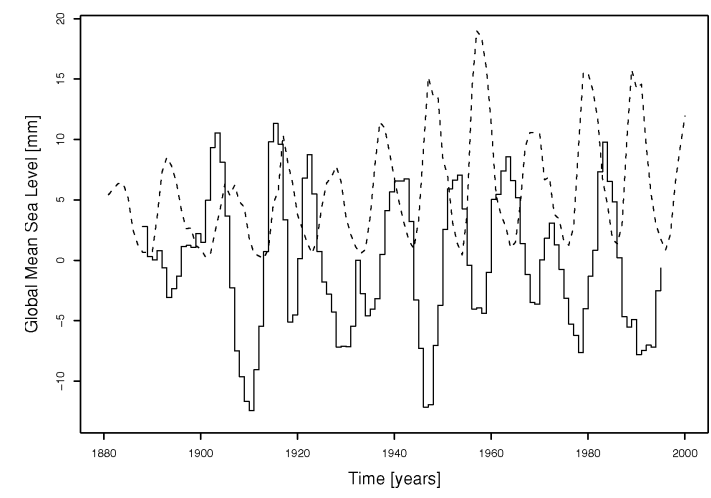

Figure 3. Detrended 3RN Global Mean Sea Level (solid) vs. Solar-spot Cycle (dash).

\section{References}

Bruckman, W. \& Ramos, E. 2003, Submitted to Geophys. Res. Lett. (unpublished).

Bruckman, W. \& Ramos, E. 2003, Simposio: Cambio Climatico: Universidad de Puerto Rico(UPR) Bayamon. Proceedings UPR-Bayamon Library

Bruckman, W. \& Ramos, E. 2007, Congreso Internacional Gaia y El Cambio Climatico 2007, UPR-Rio Piedras, PR. Revista Umbral,Primer tomo

Eddy, J. A. 1976, Science, 192, 1189.

Haigh, J. D. 1999, Quart. J. Roy. Meteor. Soc., 125, 871-892.

Haigh, J. D. 1999, J. Atmos. Solar Terrest. Physics, Gl, 63-72.

Haigh, J. D. 1996, Science, 272, 981-984.

Jevrejeva, S., Moore, J. C. Grinsted, A., \& Woodworth, P. L. 2008, Geophys. Res. Lett., 3, L08715.

Kniveton, D. R. \& Todd, M. C. 2001, Geophys. Res. Lett., 28, 1527-1530.

Maunder, E. W. 1890, Mon. Not. R. Astron. Soc., 50, 251.

Shindell, D., Rind, D., Balabhandran, N., Lean, J., \& Lonergan 1999, Science, 284, 305.

Sporer, F. W. 1887, Vierteljahrsschr. Astron. Ges. Leipzig, 22, 323.

Svensmark, H. \& Friis-Christensen, E. 1997, J. Atm. Sol. Terr. Phys, 284, 59, 1225-1232.

Svensmark, H. \& Friis-Christensen, E. 1998, Phys. Rev. Lett., 81, 5027-5030.

Wolf, R. 1856, Astron. Mitt. Zurich, 1, VIII.

Wolf, R. 1868, Astron. Mitt: Zurich, 24, III. 